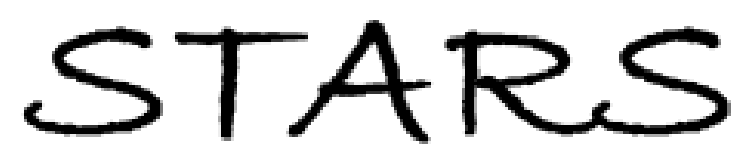

University of Central Florida

STARS

Faculty Bibliography 1990s

Faculty Bibliography

$1-1-1998$

\title{
Evidence for self-mode-locking in p-Ge laser emission
}

\author{
A. V. Muravjov \\ University of Central Florida \\ R. C. Strijbos \\ University of Central Florida \\ C. J. Fredricksen \\ University of Central Florida \\ H. Weidner \\ University of Central Florida \\ W. Trimble \\ University of Central Florida
}

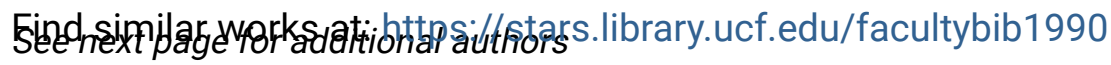

University of Central Florida Libraries http://library.ucf.edu

This Article is brought to you for free and open access by the Faculty Bibliography at STARS. It has been accepted for inclusion in Faculty Bibliography 1990s by an authorized administrator of STARS. For more information, please contactSTARS@ucf.edu.

\section{Recommended Citation}

Muravjov, A. V.; Strijbos, R. C.; Fredricksen, C. J.; Weidner, H.; Trimble, W.; Withers, S. H.; Pavlov, S. G.; Shastin, V. N.; and Peale, R. E., "Evidence for self-mode-locking in p-Ge laser emission" (1998). Faculty Bibliography 1990s. 2376.

https://stars.library.ucf.edu/facultybib1990/2376

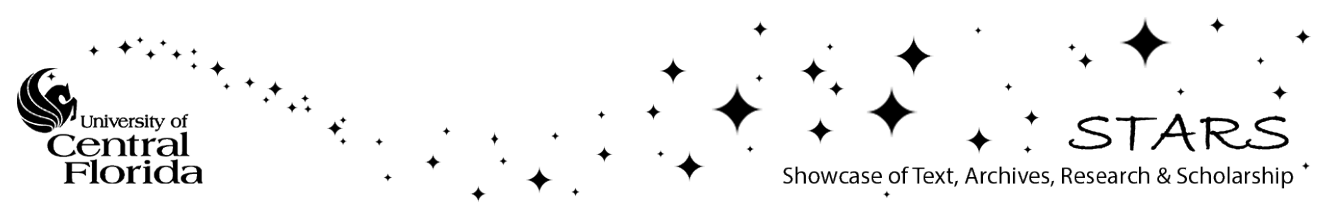




\section{Authors}

A. V. Muravjov, R. C. Strijbos, C. J. Fredricksen, H. Weidner, W. Trimble, S. H. Withers, S. G. Pavlov, V. N. Shastin, and R. E. Peale 


\section{Evidence for self-mode-locking in p-Ge laser emission}

Cite as: Appl. Phys. Lett. 73, 3037 (1998); https://doi.org/10.1063/1.122664

Submitted: 08 June 1998 . Accepted: 21 September 1998 . Published Online: 19 November 1998

A. V. Muravjov, R. C. Strijbos, C. J. Fredricksen, H. Weidner, W. Trimble, S. H. Withers, S. G. Pavlov, V. N. Shastin, and R. E. Peale

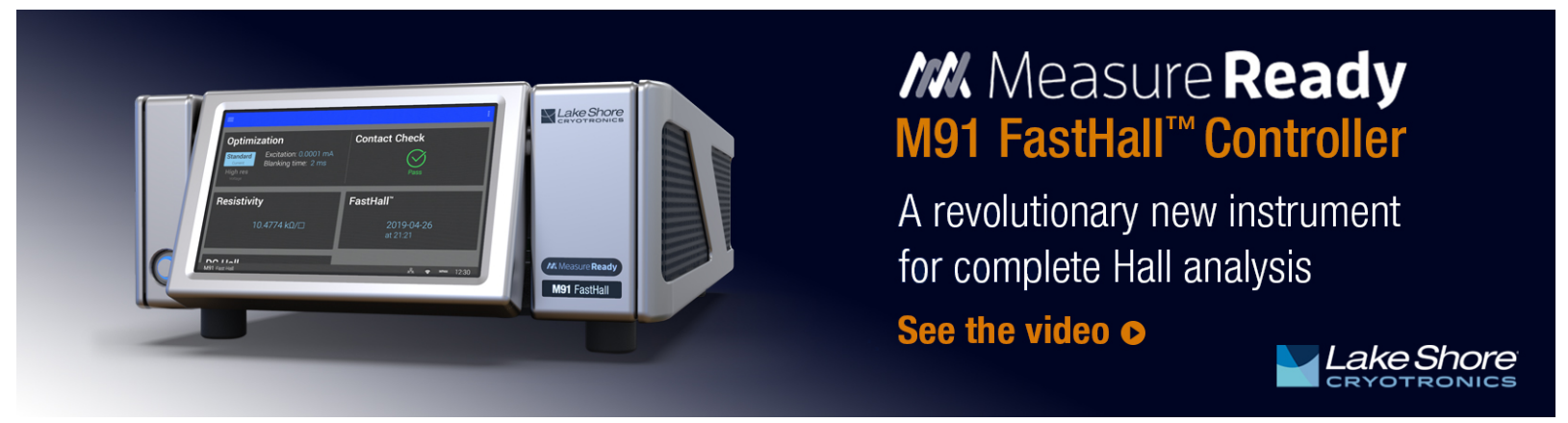




\title{
Evidence for self-mode-locking in $\boldsymbol{p}$-Ge laser emission
}

\author{
A. V. Muravjov, R. C. Strijbos, C. J. Fredricksen, H. Weidner, W. Trimble, \\ and S. H. Withers \\ Department of Physics, University of Central Florida, Orlando, Florida 32816
}

S. G. Pavlov and V. N. Shastin

Institute of Physics of Microstructures, Russia Academy of Sciences, GSP-105, Nizhny Novgorod 603600, Russia

R. E. Peale ${ }^{\mathrm{a})}$

Department of Physics, University of Central Florida, Orlando, Florida 32816

(Received 8 June 1998; accepted for publication 21 September 1998)

Investigations of the dynamics of the far-infrared $p$-Ge laser emission reveal strong periodic soliton-like intensity spikes with less than $100 \mathrm{ps}$ duration. We interpret these spikes as self-mode-locking of $p$-Ge laser modes. The effect becomes more pronounced when a GaAs/ $\mathrm{AlGaAs} / \mathrm{InGaAs}$ quantum well structure on a semi-insulating GaAs substrate is inserted into the laser cavity. (C) 1998 American Institute of Physics. [S0003-6951(98)01647-7]

Far-infrared $p$-Ge lasers operate in the spectral range $50-140 \mathrm{~cm}^{-1}$. Their mechanism of stimulated emission is based on direct optical transitions between light and heavy hole valence subbands in bulk $p$-Ge [Fig. 1(a)] in strong crossed electric and magnetic fields [Fig. 1(b)] when the crystal is cooled to liquid helium temperatures. The population inversion builds up for certain ratios of electric and magnetic fields, where light holes are accumulated on closed trajectories below the optical phonon energy, while heavy holes experience strong optical phonon scattering. The gain spectrum is very broad because lasing transitions lie in a wide range of hole momenta [Fig. 1(a)]. Far-infrared laser emission with a few $\mu$ s pulse durations and peak powers of 1-10 Watt are widely reported. ${ }^{1}$ Figure 2 shows the laser operating zone of applied $E$ and $B$ fields, which splits into low and high field regions. These correspond to low and high frequency laser emission, respectively.

Due to their wide spectrum $p$-Ge lasers are promising for generation of short (picosecond) pulses of $\mathrm{THz}$ radiation by means of active $\mathrm{e}^{2-4}$ or passive mode-locking for timeresolved (linear and nonlinear) $\mathrm{THz}$ spectroscopy and other related applications. In Refs. 2-4 200 ps pulses of $p$-Ge laser radiation with a considerable power have been obtained by active mode locking, when the gain is externally modulated at the laser cavity roundtrip frequency. Without active mode locking, the laser still might have a strongly varying intensity, periodic with the roundtrip time of the laser cavity, ${ }^{3,5}$ which results from modes being accidentally in phase to some extent. For the low frequency part of the laser spectrum, which is split into several discrete lines containing only 10 to 20 longitudinal modes (Fig. 3), such coincidences are likely, especially in the rising edge of the laser pulse, and this has been interpreted in Ref. 3 as self-mode-locking. In the high frequency part of the spectrum, hundreds of modes oscillate simultaneously with random phases, and a sharply

${ }^{\text {a)}}$ Electronic mail: rep@physics.ucf.edu peaked temporal structure due to some coincidental phase relation is much more unlikely.

In this letter the first evidence for self-mode locking in the high frequency region of the $p$-Ge laser is reported, appearing as a clear nonlinear effect at the peaks of a slowly oscillating laser intensity. As a result, a train of radiation spikes shorter than $100 \mathrm{ps}$ is generated without active gain modulation or added passive elements.

The active Ga-doped Ge crystal $N_{A} \simeq 7 \times 10^{13} \mathrm{~cm}^{-3}$ has dimensions $4.5 \times 7.3 \times 28.0 \mathrm{~mm}^{3}$. The crystal ends were polished flat and parallel to each other within $1 \mathrm{~min}$ accuracy, and two external copper mirrors were attached to them insulated from the Ge and the aluminum contacts with $20 \mu \mathrm{m}$ teflon film [Fig. 1(b)]. The crystal is cooled by immersion in liquid helium at $4 \mathrm{~K}$. Electric field pulses $(2 \mu \mathrm{s})$ are applied along [110] via ohmic contacts covering the $4.5 \times 28.0 \mathrm{~mm}^{2}$ surfaces of the sample. The magnetic field is applied along $[1 \overline{1} 0]$ in Faraday geometry. The laser radiation propagates along the long crystal axis [1 $1 \overline{0} 0]$, is conducted out of the cryostat, is detected after a teflon lens with a fast whiskercontacted Schottky diode [1T17(82), University of Virginia], amplified with a $10 \mathrm{GHz}$ bandwidth amplifier (Picosecond Pulse Labs 5840) and recorded on a transient digitizer (Tektronix SCD5000) with 80 ps resolution. Spectroscopy was

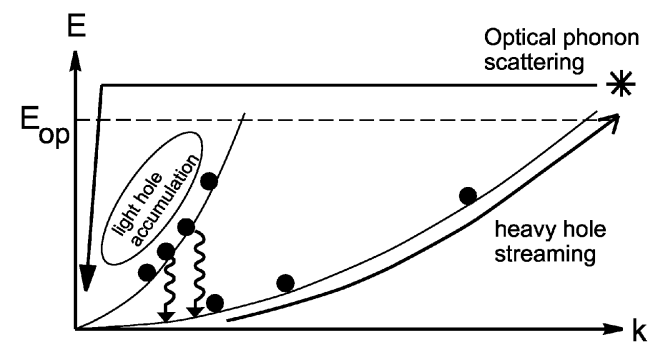

(a)

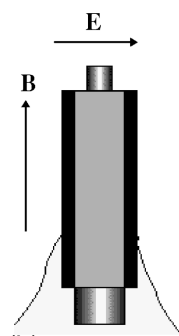

(b)

FIG. 1. (a) Pumping mechanism of the $p$-Ge laser. (b) Laser cavity construction. The black strips indicate Al coated lateral sides, with wires indium-soldered to them. The cylindrical end pieces are $\mathrm{Cu}$ mirrors, insulated from the crystal with $20 \mu \mathrm{m}$ teflon film. 


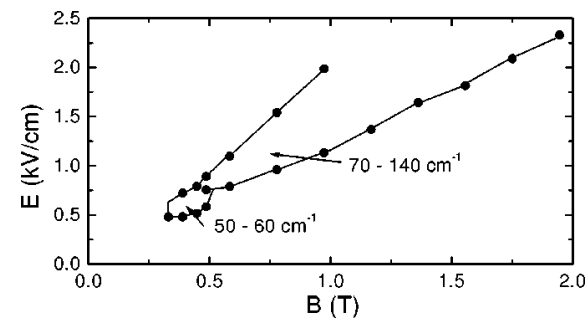

FIG. 2. Typical $(E, B)$ field regions for low- and high-frequency operation of the $p$-Ge laser.

performed with a research grade Fourier transform infrared (FTIR) spectrometer (Bomem DA8) using "event-locked" gated-acquisition electronics ${ }^{6}$ (Zaubertek).

The $p$-Ge laser spectrum without intracavity frequency selection is $15-30 \mathrm{~cm}^{-1}$ broad and tunable between 50 and $140 \mathrm{~cm}^{-1}$ by changing the applied fields. ${ }^{1}$ In the highfrequency region (Fig. 2), this spectrum can contain a few hundred longitudinal laser modes, but in the low-frequency region below $70 \mathrm{~cm}^{-1}$, the spectrum is split into discrete lines related to shallow impurity transitions. ${ }^{7}$ The broadest of the low frequency laser emission lines is shown in Fig. 3. This is the first broadband spectral measurement of $p$-Ge laser output with resolution high enough to resolve the longitudinal mode structure. The observed equidistant mode spacing $\Delta \nu$ in Fig. 3 (in $\mathrm{Hz}$ ) is related to the longitudinal round-trip time $\tau$ by $\Delta \nu=1 / \tau=c / 2 n_{\mathrm{Ge}} L=1363 \mathrm{MHz}$, or $\tau$ $=734 \mathrm{ps}$, where $L$ is the active crystal length, and $n_{\mathrm{Ge}}$ $=3.925$ is the refractive index. The corresponding mode beating pattern could easily display strong oscillations of the laser intensity at the round-trip frequency $\Delta \nu$, simply by having the phases of each mode accidentally similar. In the high field region, however, such coincidences and strong oscillations are much less likely.

Figure 4 shows digitally captured traces of the output intensity of this particular crystal in the high field region at three different time scales. The output intensity is strongly modulated at about $44 \mathrm{MHz}$ [Fig. 4(a)]. These oscillations are much slower than the cavity round-trip frequency of $1363 \mathrm{MHz}$. At a higher temporal resolution, trains of very sharp spikes are found on the maxima of the slow intensity oscillations [Fig. 4(b)]. Such spikes are absent from the 44 $\mathrm{MHz}$ valleys and they start to grow rapidly as the intensity increases. The round trip time for the sharp spikes is $722 \mathrm{ps}$, which is close to the calculated cavity round-trip time of 734 ps. We take the occurrence of the sharp spikes to be evidence for self-mode-locking. Figures 4(c) and 4(d) show close-ups of portions of the trace in Fig. 4(b). Sampled data points are

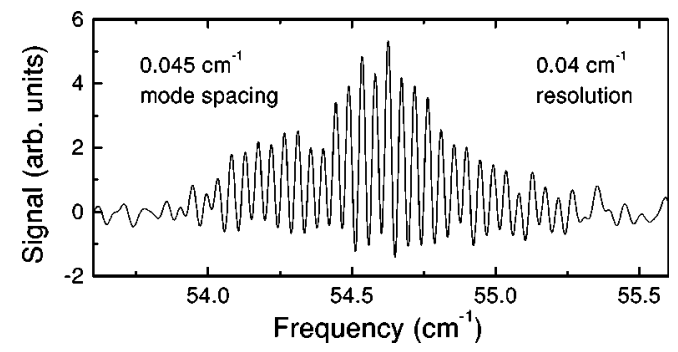

FIG. 3. Close-up of one of the spectral lines in the low-field regime showing longitudinal mode structure.
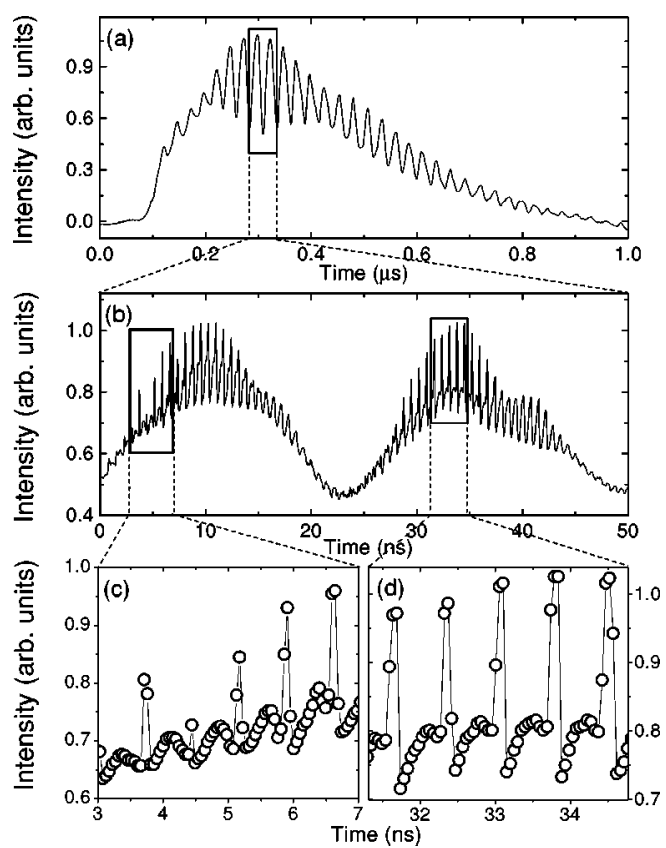

FIG. 4. (a) Laser output pulse showing $44 \mathrm{MHz}$ oscillations. (b) A different shot at higher time resolution showing sharp spikes at the crests of the 44 MHz oscillations. (c) and (d) Further close-ups of (b) show that the unevenness in the peak heights is evidently a sampling effect, where points are sampled at a $50 \mathrm{ps}$ interval.

indicated by circles. The unevenness in the rise of self-mode locking in Fig. 4(c) is clearly a sampling effect. The points are separated by $50 \mathrm{ps}$, showing that the self-mode-locked pulses have a width less than the 80 ps rise time allowed by the bandwidth of the electronics. These are the shortest pulses yet observed from $p$-Ge lasers. ${ }^{2-4}$

Additionally, we have investigated the temporal behavior of emitted radiation from this $p$-Ge laser when a GaAs/ $\mathrm{AlGaAs} / \mathrm{InGaAs}$ quantum well structure on a semi-insulating GaAs substrate with a thickness of $0.5 \mathrm{~mm}$ and parallel polished surfaces is inserted into the cavity between the active crystal and the back mirror. The short radiation spikes become much more pronounced and repeatable. This is shown in Fig. 5. The inset shows another shot at a finer timescale. The quantum well structure consists of $40 \mathrm{~nm} n^{+}$-GaAs $(n$ $\left.>10^{18} \mathrm{~cm}^{-3}\right), 40 \mathrm{~nm} n^{+}-\mathrm{Al}_{0.24} \mathrm{Ga}_{0.76} \mathrm{As}\left(n>10^{18} \mathrm{~cm}^{-3}\right)$, $15 \mathrm{~nm} i-\mathrm{In}_{0.18} \mathrm{Ga}_{0.82} \mathrm{As}$, and $100 \mathrm{~nm} i$-GaAs layers on top of a GaAs substrate.

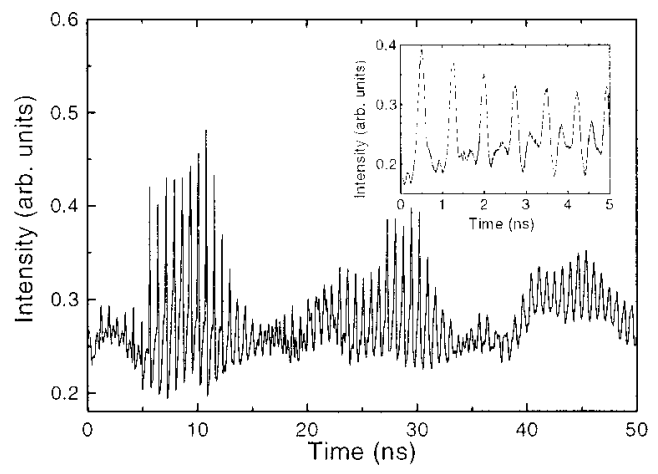

FIG. 5. Output of the self-mode-locked $p$-Ge laser with quantum well intracavity insert. Upon blocking the radiation, the signal drops to a baseline of about 0.05 , so the peaks occur on a constant background of about equal intensity. The inset shows a different shot with higher time resolution. 
Thus these experiments demonstrate self-generation of intense spikes as short as $80 \mathrm{ps}$ in the far-infrared $p$-Ge laser, which is shorter than obtained so far by active mode locking. ${ }^{2-4}$ We take this result as tentative evidence for selfmode-locking. Strong slow intensity oscillations seem to provide the conditions for buildup of these spikes. The experimental data are not yet conclusive with respect to the origin of either the slow oscillations or the short pulse formation. Various possibilities are discussed here briefly.

The most straightforward explanation for the $44 \mathrm{MHz}$ oscillation is beating of transverse modes ${ }^{8}$ and this was in fact suggested in Ref. 9 to explain weak $50 \mathrm{MHz}$ oscillations in the output of a $p$-Ge laser. The observed synchronization of spike generation to the rise in intensity might, however, suggest that actual modulation of the gain occurs at this low frequency, and peaks grow during a period of net gain and disappear when losses are dominant. The observed train of spikes corresponds to the formation of a soliton-like electromagnetic pulse traveling in the laser cavity. Since the active $p$-Ge laser crystal is a hot carrier system interacting with a high-intensity radiative field and operating in a strongly nonlinear regime in many aspects, several possible mechanisms can be suggested for self-mode locking. First of all, the population inversion in part of the crystal might be destroyed due to the inhomogeneity of doping and/or inhomogeneity of the electric field. This part could then act as a saturable absorber causing passive mode locking. The observed enhancement upon including a nonlinearly absorbing spacer into the cavity seems to support such an interpretation. However, the observed rapid rise in spike intensity remains somewhat surprising, considering the supposed low gain of the medium, ${ }^{1}$ and this might suggest other mechanisms such as soliton mode locking due to nonlinear changes of the refractive index. Unfortunately, the magnitude of these index changes in $p$-Ge laser systems has been studied little so far, although the suggested strongly nonlinear birefringence caused by Faraday rotation ${ }^{10}$ and slightly different index for higherorder transverse modes, ${ }^{9}$ might turn out to be important factors for the generation of temporal ${ }^{11}$ or spatial optical solitons, respectively. ${ }^{12}$ Clearly, further experimental and theoretical studies are necessary to clarify this issue.

The passive way of obtaining picosecond far-infrared pulses from a $p$-Ge laser is much simpler than the active mode-locking scheme, which involves high-power radio frequency (rf) techniques. ${ }^{2-4}$ A disadvantage of self-modelocking is the rather large background output intensity (about 40\%-50\%) observed in Figs. 4 and 5, which is suppressed when the laser is actively mode locked with a sufficiently strong rf field. However, to the extent that this background is due to unlocked, or spatially separated (internal reflection) modes, it can possibly be removed by bringing these modes below threshold with an optimized resonator design. Also, hybrid mode-locking schemes, where active and passive mode locking are combined, could yield the advantages of both schemes: short pulses with low jitter and background. The rapid rise in pulse intensity suggests that pulse amplitude modulation telemetry with a mode-locked pulse train of $\mathrm{THz}$ laser radiation may be possible, although it remains unclear how to control such modulation. Potential applications might include secure, local area communication by $\mathrm{THz}$ beams or longer range communication in space. ${ }^{13}$

This work is supported by NSF (ECS-9531933) and AFOSR/BMDO (F49620-97-1-0434). Coauthors from IPM thank Russian Foundation for Basic Research (96-02-19275) for support of this work.

${ }^{1}$ Opt. Quantum Electronics, edited by E. Gornik and A. A. Andronov (Chapman and Hall, London, 1991), Vol. 23, Special Issue Far-infrared Semiconductor Lasers.

${ }^{2}$ J. N. Hovenier, A. V. Muravjov, S. G. Pavlov, V. N. Shastin, R. C. Strijbos, and W. Th. Wenckebach, Appl. Phys. Lett. 71, 443 (1997).

${ }^{3}$ J. N. Hovenier, T. O. Klaassen, W. Th. Wenckebach, A. V. Muravjov, S. G. Pavlov, and V. N. Shastin, Appl. Phys. Lett. 72, 1140 (1998).

${ }^{4}$ A. V. Muravjov, R. C. Strijbos, C. J. Fredricksen, H. Weidner, W. Trimble, A. Jamison, S. G. Pavlov, V. N. Shastin, and R. E. Peale, in Proceedings of Workshop on Radiative Processes and Dephasing in Semiconductors, February 2-4, 1998, Coeur d'Alene, Idaho, OSA-TOPS, Vol. 18 edited by D. Citron (OSA, Washington DC, 1998), pp. 102-107.

${ }^{5}$ A. V. Muravjov, R. C. Strijbos, C. J. Fredricksen, H. Weidner, W. Trimble, A. Jamison, S. G. Pavlov, V. N. Shastin, and R. E. Peale, in Conference on Lasers and Electro-Optics, 1998 OSA Technical Digest Series, Vol. 6 (Optical Society of America, Washington DC, 1998), pp. 132-133.

${ }^{6}$ H. Weidner and R. E. Peale, Appl. Spectrosc. 51, 1106 (1997), Zaubertek, Inc., 1007 Silcox Branch Circle, Oviedo, FL 32765.

${ }^{7}$ S. V. Demihovsky, A. V. Murav'ev, S. G. Pavlov, and V. N. Shastin, Semicond. Sci. Technol. 7, B622 (1992).

${ }^{8}$ A. E. Siegman, Lasers (University Science Books, Mill Valley, California, 1986).

${ }^{9}$ A. V. Bespalov, Appl. Phys. Lett. 66, 2703 (1995).

${ }^{10}$ S. Komiyama, S. Kuroda, and T. Yamamoto, J. Appl. Phys. 62, 3552 (1987).

${ }^{11}$ S. Wabnitz, E. Westin, R. Frey, and C. Flytzanis, J. Opt. Soc. Am. B 13, 2420 (1996).

${ }^{12}$ Compact Sources of Ultrashort Pulses, Cambridge Studies in Modern Optics, edited by I. N. Duling III (Cambridge University Press, Cambridge, UK, 1995).

${ }^{13}$ New Directions in Terahertz Technology, NATO ASI Series, series E, Vol. 334 edited by J. M. Chamberlain and R. E. Miles (Kluwer, Boston, 1997). 